\title{
Über die Abhängigkeit des Aktionsstroms des Ureters von der Reizstärke.
}

\author{
Von \\ Masatake Yano. \\ (矢野正武) \\ (Aus dem II. Institut der Physiologie der Tohoku Reichsuni- \\ versität zu Sendai, Vorstand: Prof. Dr. K. Motokawa)
}

\section{Einleitung.}

Orbeli und Brücke, 1910, führten Forschungsarbeiten über den Aktionsstrom des Ureters durch. Doch dies Problem ist seitdem lange vernachlässigt worden, und diesbezügliche wenige Mitteilungen waren meistens auf den Aktionsstrom bei der spontanen Kontraktion beschränkt. Ich ${ }^{1)}$ habe in der vorausgegangenen Arbeit nun festgestellt, dass die Kontraktion des Schweineureters unter guten physiologischen Bedingungen dem Alles oder Nichts-Gesetz folgt.

Es gilt nun zu untersuchen, wie sich auch der Aktionsstrom diesem Gesetz gegenüber verhält.

\section{Methode.}

Um den abpräparierten Ureter möglichst in physiologischem Zustand zu erhalten und die Reizung und $\mathrm{Ab}$ leitung des Aktionsstroms zu erleichtern, benutzte ich ein Glasgefäss, das durch vier Scheidewände aus Zelluloid in fünf Zimmerchen geteilt war (Fig. 1 a).

Alle Scheidewände waren mit je einem Loch versehen,

Fig. 1. Versuchsanordnung. $e_{1}, e_{2}$ : Reizelektroden. $e_{3}, e_{4}$ : Ableitelektroden.

G: Galvanometer.

U: Ureter.

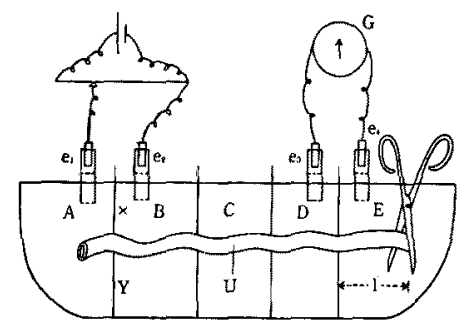

a



b 
so dass sich der Ureter durch diese Löcher hindurch entlang der Längsachse des Gefässes erstreckte. Jede Zelluloidscheidewand bestand aus zwei Teilen $X$ und $Y$, wie in Fig. 1 b gezeigt wird. Wenn man die $X$ Platte in die Y-Platte hineinsteckte, konnte man die Grösse des Loches je nach dem Steckungsgrad beliebig regulieren, so dass es sich dem Umfang des Ureters gut anpasste. Am Beginn des Versuchs legte man den ausgeschnittenen Schweineureter gerade auf die Vertiefungen der Y-Platten, dann wurden die X-Platten angepasst. Dies Verfahren genügte den Verkehr der Tyrodeschen Lösung der nebeneinander liegenden Zimmerchen abzusperren, und zwar störte das niemals die Erregungsleitung am Ureter. Um der Sauerstoffzufuhr sicher zu sein, wurden immer Luftbläschen in die Tyrodesche Lösung in den Zimmerchen gesandt. Die Zimmerchen des Gefässes seien einfachheitshalber von links nach rechts als A, B, C, D und E grezeichnet.

Die Reizapparate sind dieselben wie in der vorigen Arbeit ${ }^{1)}$, abgesehen davon, dass die Gelatinelektroden statt der unpolarisierbaren Fadenelektroden angewandt wurden. Die Anode der Reizelektroden wurde in $A$, die Kathode in $B$, und die Ableitungselektroden $e_{3}$ und $\mathrm{e}_{4}$ wurden in D und $\mathrm{E}$ respektiv eingetaucht. Das Zimmerchen $\mathrm{C}$, in welchem keine Elektrode gesetzt war, stellte das Verbindungsstuick zwischen dem Reiz- und dem Ableitungsort dar und war für die Vorbeugung der Stromschleife notwendig.

Die Empfindlichkeit des Galvanometers (Yokokawa) war 2,1 $\times$ $10^{-9}$ A, $6 \times 10^{-6} \mathrm{~V}$, sein innerer Widerstand betrug $300 \Omega$ und seine Periode 4 Sekunden. Die Periode dieses Galvanometers ist nicht immer ausreichend, um den Aktionsstrom des Ureters naturgetreu zu registrieren und daher kann vom naturgetreuen Verlauf desselben bei den mit diesem Instrument registrierten Kurven natürlich keine Rede sein, aber es wird genügen für den Vergleich der Grösse des Aktionsstroms, welcher das Hauptthema vorliegender Arbeit darstellt.

\section{Ergebnisse.}

Der A ktionsstrom des Ureters ist diphasisch, wenn ein Schnittende des Ureters im Zimmerchen $\mathbf{E}$ vorliegt. Wenn man diesen in $\mathrm{E}$ gelegenen Teil vom Ende stückweise abschneidet, so verändert sich die Form des Aktionsstroms je nach der Länge des Ureterrestes in $\mathbf{E}$ derart, dass sich der Ausschlag nach oben $\left(e_{3}\right.$ negativ gegen $\left.e_{4}\right)$ auf Kosten des nach unten gerichteten Nachschlags vergrössert. Der letztere verkleinert sich aber nicht stetig mit der Abkürzung des in E gelegenen Teils, sondern vergrössert sich vorübergehend, um darauf allmählich 
bis zum Verschwinden abzunehmen. Dies Verhältnis ist in Fig. 2 und Tabelle I dargestellt.

Fig. 2. Abhängigkeit der Aktionsstromgrösse von der Restlänge (1) des Ureters im Kammerchen E.

Zeitmarke 1 Sek.

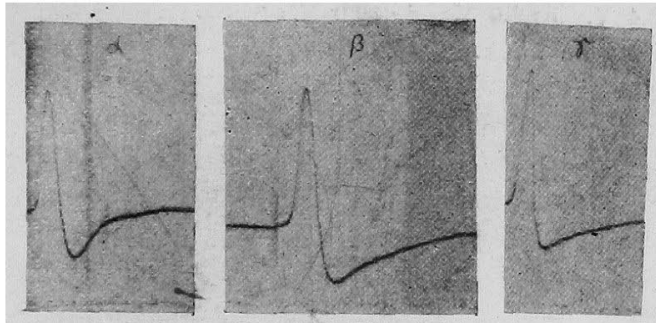

Tabelle I.

Die Messung der Fig..2.

\begin{tabular}{c|c|c|c}
\hline & $\begin{array}{c}\text { Länge des } \\
\text { Ureterteils } \\
\text { in E (1) }\end{array}$ & $\begin{array}{c}\text { Ausschlag } \\
\text { nach oben }\end{array}$ & $\begin{array}{c}\text { Ausschlag } \\
\text { nach unten }\end{array}$ \\
\hline$\alpha$ & $40 \mathrm{~mm}$ & $25,0 \mathrm{~mm}$ & $9,3 \mathrm{~mm}$ \\
$\beta$ & 20 & 28,5 & 11,0 \\
$\gamma$ & 10 & 28,3 & 8,0 \\
$\delta$ & 5 & 32,0 & 0 \\
$\varepsilon$ & 0 & 37,0 & 0
\end{tabular}

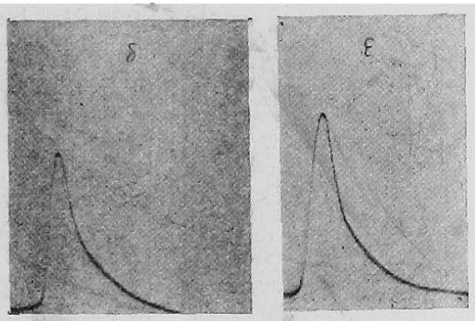

Bozler 2) (Katze, Maus, ausgeschnittener Ureter) konnte den monophasischen Aktionsstrom durch die Kneifung des Ureterteils in der Nähe einer Ableitelektrode ableiten, wobei der Ausschlag nach oben unverändert blieb.

Dieser Befund widerspricht

dem meinigen, denn der Ausschlag nach oben des monophasischen Aktionsstroms ist bei meiner Untersuchung immer grösser als beim biphasischen.

Wenn der Teil in E restlos abgeschnitten wurde, war der Aktionsstrom nicht immer rein monophasisch, sondern zeitweise liessen sich

Fig. 3. Nebenschwankung vor und nach der Hauptschwankung.

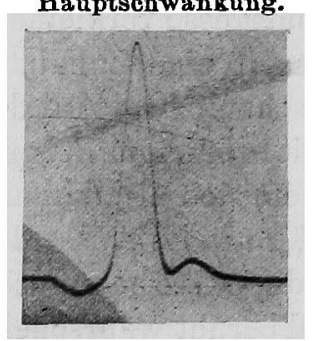

Nebenschwankungen vor oder nach der Hauptschw̉ankung bemerken (Fig. 3). Derartige Schwankungen sind auch von verschiedenen Forschern wie Hasama ${ }^{3)}, \mathrm{Aiba}^{4)}$ und Kon ${ }^{5)}$ beobachtet worden, aber über den Entstehungsmechanismus sind ihre Meinungen nicht ganz übereinstimmend, so dass es noch weiterer Forschungen darüber bedarf.

Ich bestimmte die Schwelle für den monophasischen Aktionsstrom durch die Abstufung je $1 \mathrm{~mm}$ in dem $100 \mathrm{~cm}$ langen Brückendrahte und registrierte Aktionsströme durch überschwellige Reizung von verschiedener Stärke. Aus dieser Untersuchung hat sich herausgestellt, dass die Grösse 
des Aktionsstroms ungeachtet der Reizstärke stets gleich gross ist (Fig. 4 u. Tab. II). Dieses Resultat stimmt mit meinem Ergebnis über die Kontraktion des Ureters völlig überein.

Fig. 4. Unabhängigkeit der Aktionsstromgrösse von der Reizstärke. Reizstärke (relative Werte)

a : 1,968 V. b:2,4 V. c : 6,0 V. d: 12,0 V.

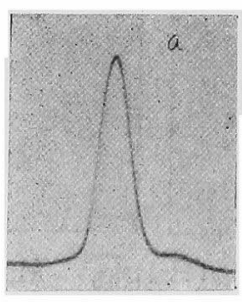

Tabelle II.

Die Messung der Fig. 4.

\begin{tabular}{c|c|c}
\hline & Reizstärke (V) & $\begin{array}{c}\text { Grösse des } \\
\text { Ausschlages } \\
\text { in mm }\end{array}$ \\
\hline & 1,956 & 0 \\
a & 1,968 & 44,4 \\
b & 2,400 & 43,2 \\
c & 6,000 & 42,0 \\
d & 12,000 & 41,0
\end{tabular}

Zeitmarke 1 Sek.
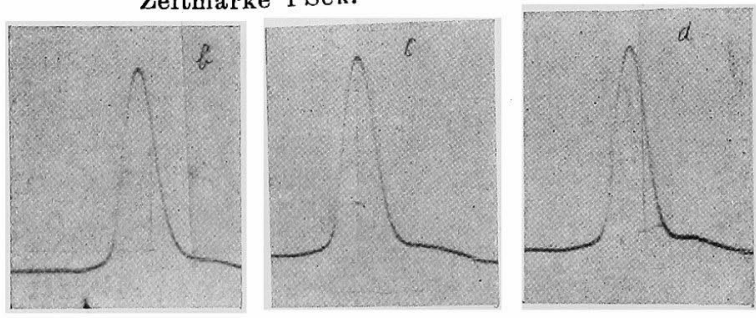

Auch Aiba gab an, dass die Grösse des diphasischen Aktionsstroms vom Ureter des Kaninchens in situ dem Alles oder Nichts-Gesetz folgt, wenn auch die Schwellenbestimmung bei seiner Untersuchung nicht so genau ausgeführt wurde. Bozler zog aus seinem Versuche denselben Schluss, liess aber dabei die

Reizstärke, insbesondere die Schwelle und den maximalen Reiz beinahe unberührt.

Weiter untersuchte ich das Verhalten der Aktionsströme gegen doppelte Reizung. Die Grösse des durch die zweite Reizung hervorgerufenen Aktionsstroms ist je nach dem Reizintervall verschieden, und zwar desto kleiner, je kürzer das Reizintervall ist. Im relativen Refraktärstadium wird nicht nur die Aktionsstromgrösse verkleinert, sondern erfahren auch die Latenz und Leitungszeit eine Zunahme. Dieses Verhältnis ist in Fig. 5 und Tab. III veranschaulicht. Auf den ersten Blick scheint die erwähnte Gesetzmässigkeit bei diesem Beispiel zu versagen, denn der zweite Ausschlag verkleinert sich nicht immer mit der Verkürzung des Reizintervalls, z. B. ist der zweite Ausschlag beim Intervall von 40 Sekunden etwas kleiner als derjenige beim Intervall von 30 Sekunden (Tab. III a). Dieser Umstand beruht wahrscheinlich auf der fortschreitenden Ermündung des Präparates. Berechnet man das Verhälttnis des zweiten Ausschlags zum jeweiligen ersten (II/I in Tabelle), so nimmt dies Verhältnis immer mit der Verkleinerung des 
Fig. 5. Verhalten der Aktionsströme bei Doppelreizung.

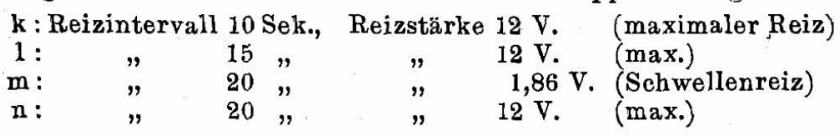
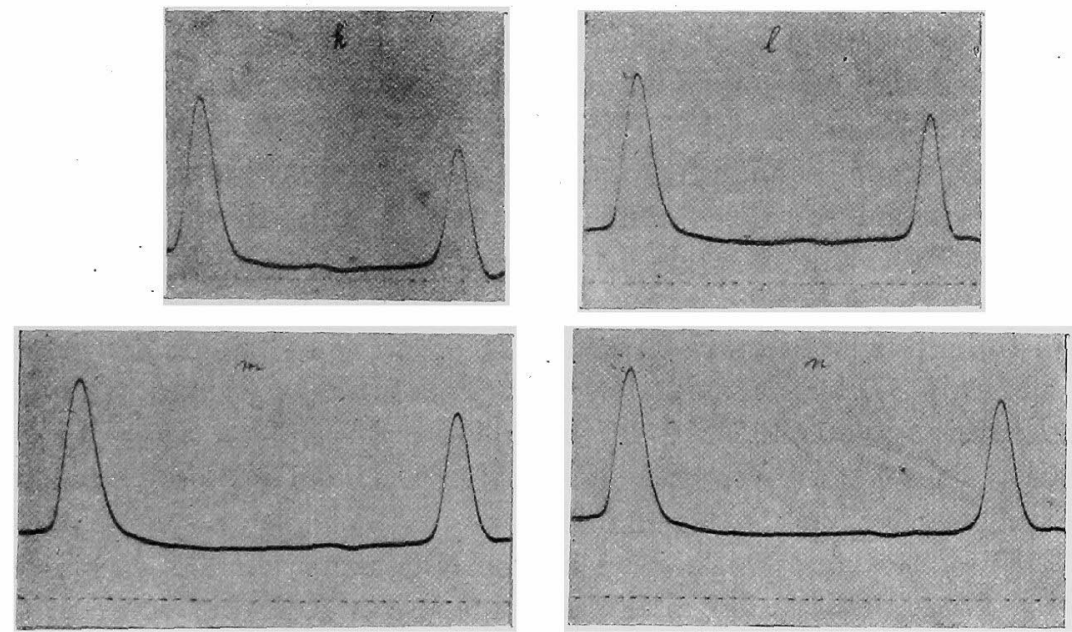

Tabelle III.

Die Abhängigkeit der Aktionsstromgrösse von der Reizstärke.

(a) Bei maximaler Reizung. Reizstärke $12 \mathrm{~V}$.

\begin{tabular}{|c|c|c|c|c|}
\hline Zeit in Min & Reizintervall in Sek. & Ausschlag $I$ in $\mathrm{mm}$ & Ausschlag II in $\mathrm{mm}$ & $\mathrm{II} / \mathrm{I}$ \\
\hline $\begin{array}{r}0 \\
5 \\
15 \\
25 \\
35\end{array}$ & $\begin{array}{l}10 \\
15 \\
20 \\
30 \\
40\end{array}$ & $\begin{array}{l}32,3 \\
33,0 \\
31,5 \\
30,0 \\
28,5\end{array}$ & $\begin{array}{l}25,1 \\
26,5 \\
27,2 \\
27,0 \\
26,4\end{array}$ & $\begin{array}{l}0,778 \\
0,803 \\
0,862 \\
0,900 \\
0,931\end{array}$ \\
\hline 35 & (b) Bei Schwell & reizung. Reizstärk & $1,920 \mathrm{~V}$. & \\
\hline $\begin{array}{l}10 \\
20 \\
30\end{array}$ & $\begin{array}{l}20 \\
30 \\
40\end{array}$ & $\begin{array}{l}32,0 \\
30,3 \\
29,0\end{array}$ & $\begin{array}{l}27,2 \\
27,4 \\
27,0\end{array}$ & $\begin{array}{l}0,850 \\
0,904 \\
0,931\end{array}$ \\
\hline
\end{tabular}

Reizintervalls ab, was ohne weiteres die obige Annahme berechtigt.

Wie aus Fig. 5 ersichtlich, beträgt das Zeitintervall zwischen dem ersten und dem zweiten Ausschlag 18, 22 und 26 Sekunden resp., je nach dem das Reizintervall 10,15 und 20 Sekunden resp. ist. Die Differenz zwischen diesen Zahlen z. B. $18-10=8$ Sekunden stellt die Verspätung der zweiten Erregung gegen die erste dar, und zwar nimmt diese Verspätung mit der Verkürzung des Reizintervalls zu, wie z.B. 6, 7 und 8 Sekunden beim Reizintervall 20,15 und 10 Sekunden respektiv.

Vergleicht man das Verhalten der Kontraktion bei doppelter Rei- 
zung mit demjenigen des Aktionsstroms, so kann man dazwischen einige Unterschiede erblicken; die Kontraktion auf die zweite Reizung hin verkleinert sich sehr auffallend mit der Abnahme des Reizintervalls, während diese Verkleinerung beim Aktionsstrom nicht so hochgradig und folglich nicht so steil ist.

Wenn der Aktionsstrom durch den zweiten Reiz auch in dieser Weise vom Reizintervall vielfach abhängig ist, bleibt er doch von der Reizstärke fast unabhängig; ungeachtet der Reizstärke traten immer Aktionsströme von derselben Grösse für dasselbe Reizintervall auf. Wie aus Tab. III hervorgeht, ist das Verhältnis II/I ganz gleich für das gegebene Reizintervall, mag es sich um den maximalen Reiz oder den Schwellenreiz handeln. Z. B. für das Intervall von 40 Sekunden ist das VerhäItnis II/I beim maximalen Reiz (Tab. III a) 0,931 und beim Schwellenreiz auch 0,931. Man könnte also aus dieser Untersuchung schliessen, dass der Aktionsstrom des Ureters auch bei der Doppelreizung dem Alles oder Nichts-Gesetz folgt.

Diese Gesetzmässigkeit wurde von Nakazawa und Nakajima ${ }^{6}$ beim N. ischiadicus der Kröte gefunden; das Refraktärstadium des Nerven folgt dem Alles oder Nichts-Gesetz und die Erregbarkeit wird un so kleiner, je kiirzer das Reizintervall wird.

Es ist hervorzuheben, dass der Aktionsstrom des Ureters nicht nur im Refraktärstadium, sondern auch im Ermündungszustand des Präparates insofern die Alles oder Nichts-Eigenschaften aufweist, als er sich ganz unabhängig von der Reizstärke verhält.

\section{Zusammenfassung.}

1. Die Grösse des Aktionsstroms des Schweineureters folgt dem Alles oder Nichts-Gesetz.

2. BeiDoppelreizung wird die Grösse des zweiten Aktionsstroms um so kleiner, je kürzer das Reizintervall wird, aber sie ist von der Reizstärke fast unabhängig.

Zum Schluss sei es mir erlaubt, Herm Prof. Dr. K. Motokawa für seine freundliche Leitung und Unterstiitzung während dieser Arbeit meinen herzlichsten Dank auszusprechen.

\section{Literatur.}

(1) Ya no, M., Tohoku Ig. z. 1989, 25, 441.

(2) Bozlex, E., Amer. J. Physiol., 1938, 124, 508-509.

(3) Has a ma, B., Pfïgers Arch., 1933, 232, 238-245.

(4) A i ba, T., Nissin Igaku, 1933-1934, 23, 967-976; 1934, 23, 2694.

(5) Kono, K., Seirigaku Kenkyu, 1938, 15, 526.

(b) Nakazawa, K. u. K. Nakajima, Keio Igaku, 1926, 6, 1599. 